Research article

Open Access

\title{
Identification of possible candidate genes regulating Sjögren's syndrome-associated autoimmunity: a potential role for TNFSF4 in autoimmune exocrinopathy
}

\author{
Cuong Q Nguyen ${ }^{1}$, Janet G Cornelius², Lauren Cooper ${ }^{1}$, Jonathan Neff ${ }^{1}$, Joann Tao1, \\ Byung Ha Lee ${ }^{1}$ and Ammon B Peck ${ }^{1,2,3}$
}

\author{
1Department of Oral Biology, University of Florida, 1600 SW Archer Road, Gainesville, FL 32610, USA \\ 2Department of Pathology, Immunology \& Laboratory Medicine, University of Florida, 1600 SW Archer Road, Gainesville, FL 32610, USA \\ ${ }^{3}$ Center for Orphan Autoimmune Disorders, College of Dentistry, University of Florida, 1600 SW Archer Road, Gainesville, FL 32610, USA \\ Corresponding author: Cuong Q Nguyen, Nguyen@pathology.ufl.edu
}

Received: 26 Aug 2008 Revisions requested: 23 Oct 2008 Revisions received: 27 Oct 2008 Accepted: 25 Nov 2008 Published: 25 Nov 2008

Arthritis Research \& Therapy 2008, 10:R137 (doi:10.1186/ar2560)

This article is online at: http://arthritis-research.com/content/10/6/R137

(c) 2008 Nguyen et al.; licensee BioMed Central Ltd.

This is an open access article distributed under the terms of the Creative Commons Attribution License (http://creativecommons.org/licenses/by/2.0), which permits unrestricted use, distribution, and reproduction in any medium, provided the original work is properly cited.

\begin{abstract}
Introduction Sjögren syndrome (SjS) is a systemic autoimmune disease in which an immunological attack primarily against the salivary and lacrimal glands results in the loss of acinar cell tissue and function, leading to stomatitis sicca and keratoconjunctivitis sicca. In recent years, two genetic regions, one on chromosome 1 (designated autoimmune exocrinopathy 2 or Aec2) and the second on chromosome 3 (designated autoimmune exocrinopathy 1 or Aec1) derived from nonobese diabetic (NOD) mice, have been shown to be necessary and sufficient to replicate SjS-like disease in nonsusceptible C57BL/6 mice.

Methods Starting with the SjS-susceptible C57BL/6-derived mouse, referred to as C57BL/6.NOD-Aec1Aec2, we generated a large set of recombinant inbred (RI) lines containing portions of Aec2 as a means of identifying more precisely the genetic elements of chromosome 1 responsible for disease development.

Results Disease profiling of these Rl lines has revealed that the SjS susceptibility genes of $A e c 2$ lie within a region located at approximately $79 \pm 5 \mathrm{cM}$ distal to the centromere, as defined by microsatellite markers. This chromosomal region contains several sets of genes known to correlate with various immunopathological features of $\mathrm{SjS}$ as well as disease susceptibility genes for both type 1 diabetes and systemic lupus erythematosus in mice. One gene in particular, tumor necrosis factor (ligand) superfamily member 4 (or $0 \times 40$ ligand), encoding a product whose biological functions correlate with both physiological homeostasis and immune regulations, could be a potential candidate SjS susceptibility gene.

Conclusions These new RI lines represent the first step not only in fine mapping SjS susceptibility loci but also in identifying potential candidate SjS susceptibility genes. Identification of possible candidate genes permits construction of models describing underlying molecular pathogenic mechanisms in this model of SjS and establishes a basis for construction of specific gene knockout mice.
\end{abstract}

\section{Introduction}

Sjögren syndrome (SjS) is a chronic, systemic, human autoimmune disease in which an immunological attack initially against the salivary and lacrimal glands results, respectively, in dry mouth (stomatitis sicca) and dry eye (keratoconjunctivitis sicca) disease(s) [1-3]. Despite efforts to define the genetic, environmental, and immunological bases of SjS, the underly- ing etiology of this disease remains ill defined. In attempts to better define the nature of SjS autoimmunity, a variety of mouse models exhibiting various aspects of $\mathrm{SjS}$ have been studied extensively [4]. One of the more intensively studied models of SjS is the nonobese diabetic (NOD) mouse [5-9]. Based on disease profiling of various congenic partners and gene knockout lines of NOD, we have proposed that the

Aec: autoimmune exocrinopathy; ANA: anti-nuclear autoantibody; IL: interleukin; INF- $\gamma$ : interferon-gamma; LF: lymphocytic foci; MHC: major histocompatibility complex; NOD: nonobese diabetic; PBS: phosphate-buffered saline; QTL: quantitative trait loci; RAR: retinoic acid receptor; RI: recombinant inbred; RXR: retinoid $\times$ receptor; SjS: Sjögren syndrome; SOAT-1: sterol O-acyltransferase-1; TNFSF4: tumor necrosis factor ligand superfamily member $4 ; \mathrm{T}_{\text {reg }}: \mathrm{T}$ regulatory. 
development and onset of SjS-like disease in these mice can be divided into at least three distinct consecutive phases [1019]. In phase 1, a number of aberrant physiological and biochemical activities, thought to result from a genetically based retarded salivary gland organogenesis and increased acinar cell apoptosis, occur prior to and independent of detectable autoimmunity. In phase 2, believed to result from the glandular cell injury of phase 1 , small numbers of macrophages and dendritic cells are attracted to the exocrine gland where these sentinel cells recruit $T$ and $B$ lymphocytes that form lymphocytic foci (LF), some of which histologically appear as germinal centers. In phase 3 , the onset of clinical disease as defined by salivary and lacrimal gland secretory dysfunction occurs, possibly resulting first from the production of autoantibodies that interfere with the neural-acinar cell signaling pathways and then from progressive loss of acinar cell mass hastened by the action of effector $\mathrm{T}$ cells.

A genetic predisposition for development and onset of SjS-like disease in NOD mice has also been defined. First, SjS-like disease in these mice appears independent of or only weakly associated with major histocompatibility complex $(\mathrm{MHC})$ class I and class II genes [10,20], thus mimicking SjS in humans. This can be seen by the fact that the congenic strain, NOD.B10-H $2^{b}$, in which the NOD MHC $1-A^{g 7} / d d 1$ diabetes susceptibility locus was replaced by the MHC $I-A b$ locus [20], continued to show SjS-like disease, including salivary and lacrimal gland dysfunction. Second, replacing /dd loci other than Idd1 (for example, Idd9, Idd10, and Idd13) resulted in the identification of Idd 3 on chromosome 3 and $/ d d 5$ on chromosome 1 as critical genetic regions for development of SjS-like disease in NOD mice [10]. In a reverse approach, introducing both Idd3 and Idd5 derived from NOD mice into SjS-nonsusceptible C57BL/6 mice resulted in a severe SjS-like disease, confirming the contributions of these two genetic loci to the development and onset of SjS [21]. Furthermore, the preclinical nonimmune aspects manifested in phase 1 of the disease appeared to associate with the $I d d 5$ locus (referred to as autoimmune exocrinopathy 2 or Aec2), whereas the immunological aspects of the disease manifested in phases 2 and 3 of the disease appeared to associate with $/ d d 3$ (referred to as Aec1). This recently generated mouse strain is referred to as C57BL/6.NOD-Aec1Aec2. While the pathophysiological and immunological aspects may not be linked solely to one or the other genetic region (as originally proposed [22]), the complete disease profile requires genes within both of these genetic loci.

For years, identification of candidate genes associated with autoimmune diseases such as T1D [23] or systemic lupus erythematosus [24] in animal models has been providing invaluable data on delineating the genetic components of these diseases, now translating to the human disease. These studies have formed a template for our current efforts to identify the SjS susceptibility loci and candidate genes underlying SjS which, in this respect, have lagged behind many other autoimmune diseases. Although our initial work defined the Aec1 and Aec2 genetic regions present in C57BL/6.NOD-Aec1Aec2 mice as being an approximately $48.5-\mathrm{cM}$ centromeric region on chromosome 3 and an approximately 73.3-cM telomeric region on chromosome 1, respectively [10], the size of these regions precluded identification of candidate genes. Subsequently, we shortened $A e c 1$ to an approximately 19.2-cM region in the first studied recombinant inbred (RI) line, C57BL/ 6.NOD-Aec1R01Aec2 [9]. For the present study, we generated a set of new RI lines that further demarcate the boundaries of Aec2. These new C57BL/6.NOD-Aec1Aec2R(n) RI lines identify not only a much shorter $A e c 2$ sublocus at position $79 \mathrm{cM}$ of chromosome 1, but also potential candidate SjS susceptibility genes on which to build hypothetical models that can be tested for validating possible pathogenic molecular mechanisms of SjS-like disease.

\section{Materials and methods \\ Animals}

C57BL/6.NOD-Aec1Aec2R(n) and C57BL/6.NOD$\operatorname{Aec} 1 R(n) \operatorname{Aec} 2 R(n)$ mice were generated by crossing C57BL/6.NOD-Aec1Aec2 mice with C57BL/6J mice purchased from The Jackson Laboratory (Bar Harbor, ME, USA). The F1 heterozygotes were screened for the presence of crossover events within the $A e c 1$ and/or Aec2 genetic regions by microsatellite marker genotyping. Individual mice indicating a crossover in Aec2 were bred with a C57BL/6J mouse to produce $A e c 2$ crossover heterozygous male and female offspring that were then used to produce F2 generations. Mice of the F2 generations were screened for a male and female homozygous for the crossover chromosome. Once an appropriate homozygous recombinant founder pair was identified, the RI line was maintained via a single line of descent.

All RI lines were bred and maintained under specific pathogenfree conditions in the animal facility of Animal Care Services of the University of Florida (Gainesville, FL, USA). Both male and female mice 4 to 24 weeks of age were used in the following studies. All mice received water and food ad libitum. Blood samples were collected while the mice were anesthetized with isoflurane. Euthanasia was carried out by cervical dislocation after anesthetization with isoflurane or $100 \% \mathrm{CO}_{2}$. Studies described herein were approved by the University of Florida Institutional Animal Care and Use Committee.

\section{Genotyping}

To determine the genetic status of each offspring, DNA was prepared using the DNeasy Tissue Kit (Qiagen Inc., Valencia, CA, USA) from a small tail snip taken between 2 and 4 weeks of age just prior to weaning. Each DNA sample was used as a template in polymerase chain reaction amplification with D1 mit primers covering the Aec2 genetic region. Microsatellite markers that differentiated genes derived from NOD mice from those derived from C57BL/6J mice were chosen. Primer 
sequences for the microsatellite markers were based on sequences available from The Jackson Laboratory and purchased from Integrated DNA Technologies (IDT, Coralville, IA, USA).

\section{Measurement of saliva flow rates}

To measure stimulated flow rates of saliva, individual mice were weighed and given an intraperitoneal injection of $100 \mu \mathrm{L}$ of a mixture containing isoproterenol $(0.02 \mathrm{mg} / 1 \mathrm{~mL}$ of phosphate-buffered saline [PBS] $)$ and pilocarpine $(0.05 \mathrm{mg} / 1 \mathrm{~mL}$ of PBS). Saliva was collected for 10 minutes from the oral cavity of individual mice using a micropipette starting 1 minute after injection of the secretagogue. The volume of each saliva sample was measured. The saliva samples were then frozen at $-80^{\circ} \mathrm{C}$ until analyzed.

\section{Histology}

Male and female C57BL/6.NOD-Aec1Aec2R(n) mice were euthanized at various ages as indicated in the text. Submandibular and lacrimal glands were surgically removed from each mouse and placed in 10\% phosphate-buffered formalin for 24 hours. Fixed tissues were embedded in paraffin and sectioned at 5- $\mu \mathrm{m}$ thickness. Paraffin-embedded sections were de-paraffinized by immersing in xylene, followed by dehydrating in ethanol. The tissue sections were prepared and stained with hematoxylin and eosin dye (Histology Tech Services, Inc., Gainesville, FL, USA). Stained sections were observed at $\times 100$ magnifications for glandular structure and leukocyte infiltration. To detect and determine leukocytic infiltrations in salivary and lacrimal glands, a single histological section per gland per mouse was examined by two individuals blinded to the RI lines. LF, defined as aggregates of greater than 50 leukocytes, were quantified for each section.

\section{Detection of anti-nuclear autoantibodies in the sera}

Anti-nuclear autoantibodies (ANAs) in the sera of mice were detected using an ANA screening kit (Immuno Concepts, Sacramento, CA, USA). Sera were tested at dilutions of 1:40, $1: 80$, and $1: 160$. Presented in this paper, however, are data from testing sera at 1:40 dilutions. In brief, HEp-2 fixed substrate slides were overlaid with the appropriate mouse serum. Slides were incubated for 30 minutes at room temperature in a humidified chamber. After three washes for 5 minutes with PBS, the substrate slides were covered with Alexa 594-conjugated goat anti-mouse $\operatorname{lgG}(\mathrm{H} / \mathrm{L})$ (Invitrogen Corporation, Carlsbad, CA, USA) diluted 1:50 for 30 minutes at room temperature. After three washes, nuclear fluorescence was detected by fluorescence microscopy at $\times 100$ magnification.

\section{Modeling of biological pathways using Pathway Studio}

To model biological pathways from selected genes located within the redefined $A e c 2$ genetic region, Pathway Studio version 5.0 software (Ariadne Genomics, Rockville, MD, USA) and the ResNet mammalian database were used. Functions of selected genes within the two genetic regions and known SjS- related genes were first verified from the ResNet mammalian database and then imported into Pathway Studio to visually construct molecular and biological interactions or relationships among the inputted genes.

\section{Statistical analyses}

For this study, we have standardized both saliva and tear collections based on the body weight of the individual mice in an attempt to better control comparisons. We have incorporated this for mice of the C57BL/6 genetic background because, first, disease tends to occur in the $\mathrm{C} 57 \mathrm{BL} / 6$ genetic background strains at an earlier age, often necessitating collections of saliva and tears when the mice are as young as 4 to 6 weeks of age and are less than half the size of adult mice, and, second, there are greater size differences between male and female mice during the time course studied. Statistical evaluations between saliva collections were determined by using the unpaired $t$ test generated by GraphPad InStat software (GraphPad Software, Inc., San Diego, CA, USA). A two-tailed $P$ value of less than 0.05 was considered significant.

\section{Results}

Genetic profiling of the recombinant inbred lines

From an initial mating of $\mathrm{C} 57 \mathrm{BL} / 6 \mathrm{~J}$ males with $\mathrm{C} 57 \mathrm{BL} /$ 6.NOD-Aec1Aec2 females, we identified 49 unique crossovers in Aec2 of chromosome 1, consisting of 33 lines with a single crossover in Aec2 and 16 lines with a crossover in both Aec1 and Aec2. In addition, 2 lines were established using mice with pre-existing double-crossovers in the Aec2 region (RI lines 02 and 03). During the subsequent inbreeding, we were successful in generating 39 new homozygous Aec2 RI lines. To map each genetic segment of the Aec2 region remaining within each of the 39 newly generated RI lines, we selected microsatellite markers spaced approximately 4 to 5 $\mathrm{cM}$ apart along chromosome 1. As presented in Figure 1, these new RI lines, taken together, define progressively smaller genetic segments of Aec2 derived from NOD mice and permit much finer mapping for SjS susceptibility loci. Although there are at least two regions on chromosome 1 (around positions 50 and $75 \mathrm{cM}$ ) that exhibited higher numbers of recombinant events, there do not appear to be any crossover hotspots.

\section{Disease profiling of the recombinant inbred lines}

SjS-like disease in our NOD-derived mouse lines, including C57BL/6.NOD-Aec1Aec2, is characterized generally by three criteria [4], reflecting the objective criteria used to identify SjS in humans [25]. These are (a) the loss of saliva and tear flow rates over time, (b) the presence of LF in the salivary and lacrimal glands, and (c) the presence of ANAs in sera. To determine which of the RI C57BL/6.NOD-Aec1Aec2R(n) mice develop salivary gland dysfunction, temporal changes in saliva flow rates were determined for both male and female mice at an early age ( $7 \pm 1$ weeks) and then at a later age ( $22 \pm 2$ weeks). The number of mice examined for each new RI line 


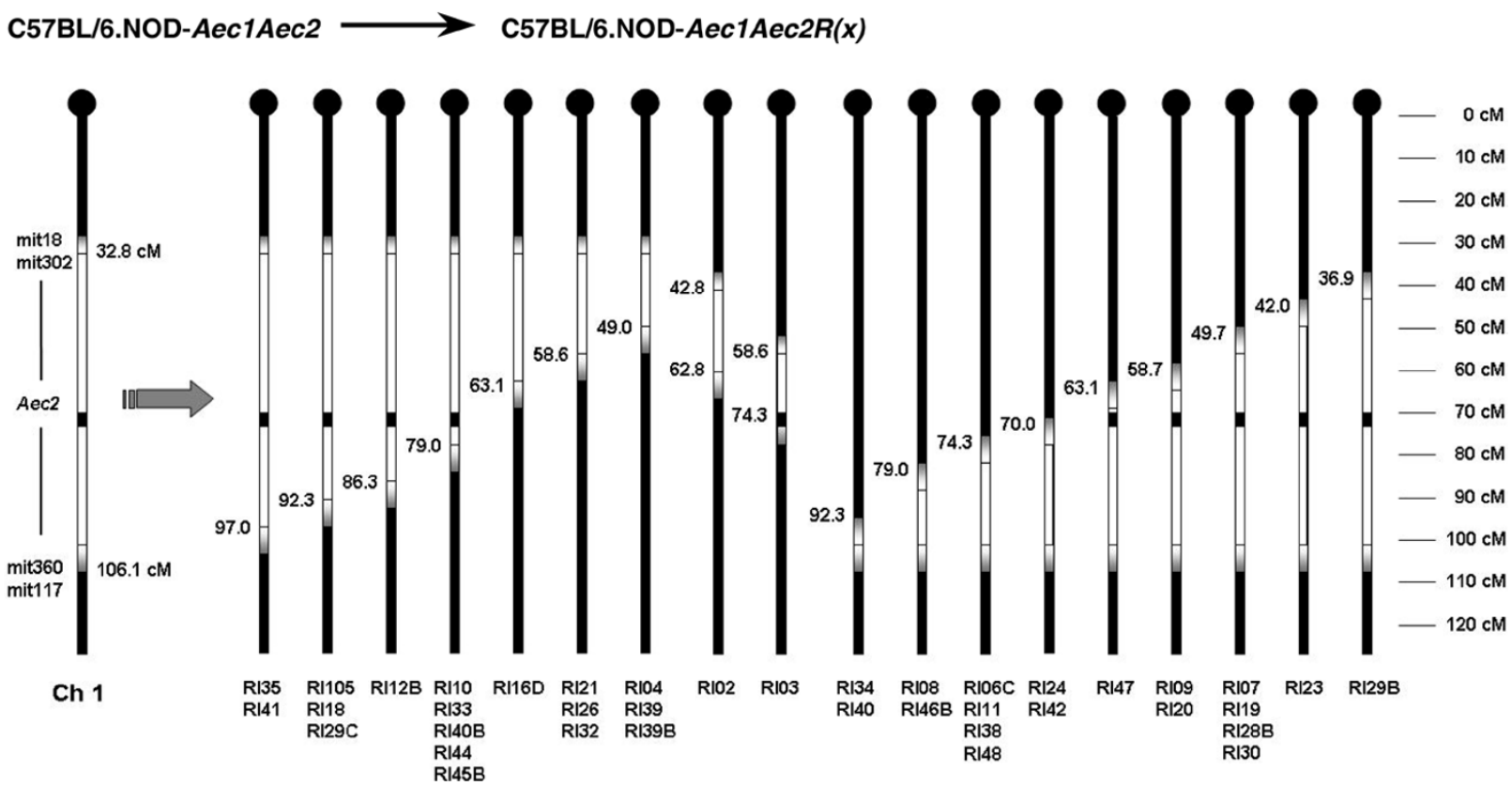

Map of chromosome 1 crossover points in C57BL/6.NOD-Aec1Aec2R(n) recombinant inbred (RI) mice. Thirty-nine RI lines are aligned to show the points of their individual crossovers in the Aec2 region of chromosome 1, as determined by D1 mit microsatellite markers. Crossover frequencies are higher at approximately $49.7,74.3$, and $79.0 \mathrm{cM}$ but are not considered hotspots for chromosomal 1 crossovers (NS: Not significant, ${ }^{*}=p<0.05$, ${ }^{\star *}=p<0.01$, and $\left.{ }^{* \star}=p<0.001\right)$.

was dependent on the number of offspring produced in the first few pregnancies following inbreeding.

Results indicate that the loss of secretory flow rates was clearly evident for several of the $\mathrm{RI}$ lines, thereby retaining the phenotype of parental C57BL/6.NOD-Aec1Aec2 mice, while a number of the RI lines also failed to show a loss of secretory activities, thereby indicating loss of the SjS-like disease phenotype. Selected yet representative data showing differences in salivary flow rates among the $A e c 2 \mathrm{RI}$ lines are shown in Figure 2. For example, both male and female mice of RI lines RI09, $\mathrm{RI} 33$, and RI12, all of which retained the parental Aec1 region but carry various portions of $A e c 2$, exhibited salivary gland dysfunction as measured by loss of salivary flow rates ranging generally between $35 \%$ and $60 \%$ as the mice aged from 8 to 20-24 weeks. These data are consistent with the decreases of saliva fluid volumes historically observed with NOD, NOD.B10-H2 ${ }^{b}$, and C57BL/6.NOD-Aec1Aec2 mice $[8,10,16,20,21]$. In contrast, male and female RI mice of lines exhibiting little or no salivary gland dysfunction (for example, RI34 and RI02) generally showed slightly increased salivary flow rates over these same time frames, mimicking SjS-nonsusceptible parental C57BL/6J mice.

Although the number of LF present in minor salivary gland biopsies of SjS patients often does not correlate directly with disease or severity of disease, both SjS patients and NODderived mice exhibiting SjS-like disease typically present with LF. As presented in Figures 3 and 4, histological examinations revealed the presence of LF in the submandibular and extraorbital lacrimal glands, starting at 8 to 12 weeks of age in all of the anticipated disease-susceptible RI strains (for example, RI06, RI09, RI33, and RI12). In contrast, no LF or at most only a relatively few, smaller LF were seen in the glands of RI34 and RI02 mice, correlating with their normal salivary flow rates. Interestingly, in addition to the lymphocytic infiltrates, increased levels of lipid deposits could be seen in the submandibular and lacrimal glands of several RI lines with onset of disease (data not shown). Quantification of LF in the salivary and lacrimal glands showing the relative differences in SjSsusceptible (RI06, RI09, RI12, and RI33) versus SjS-nonsusceptible (RI02 and RI34) RI lines is provided in Table 1.

The presence of ANAs, in particular anti-SS-A/Ro and antiSS-B/La in the sera of human patients, is one parameter in the diagnosis of clinical SjS. Concomitantly with the appearance of mononuclear leukocytes within the salivary and lacrimal glands of parental C57BL/6.NOD-Aec1Aec2 mice, increasing numbers and levels of detectable serum autoantibodies are also detected [26-29]. To identify ANAs in the sera of RI C57BL/6.NOD-Aec1Aec2R(n) mice, both male and female mice were serially bled between 6 and 24 weeks of age (until 


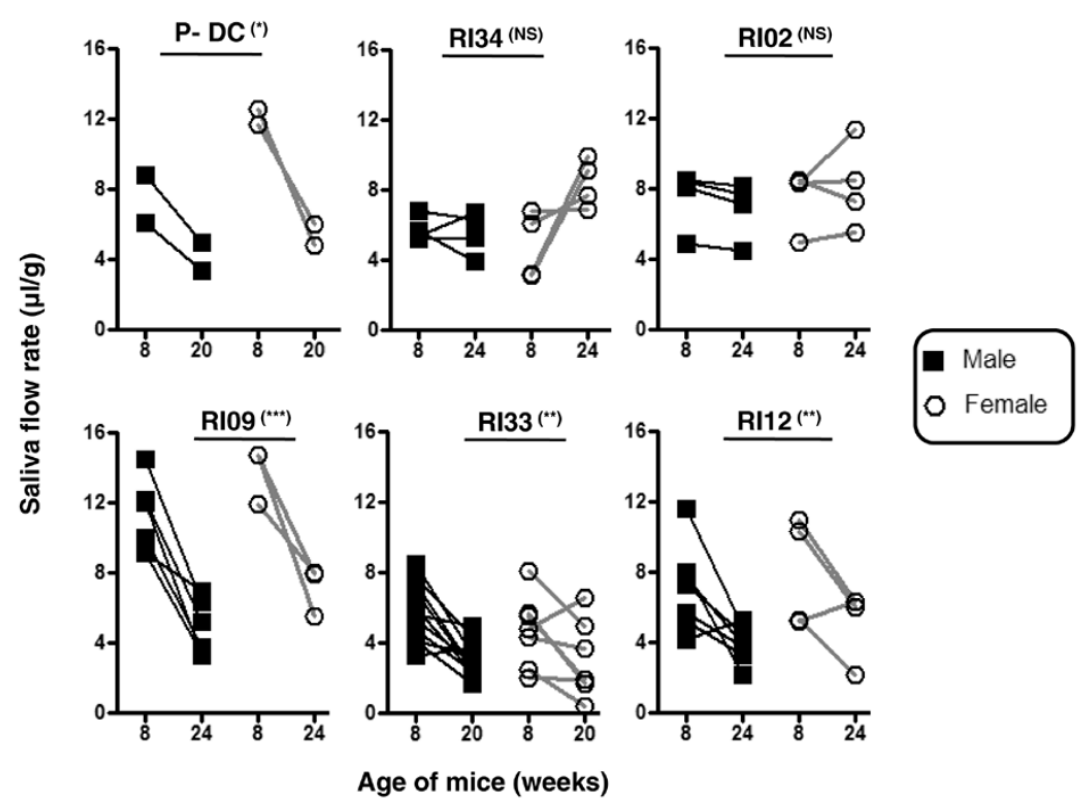

Differences in temporal loss of secretory function in various C57BL/6.NOD-Aec1Aec2R(n) mice. Male and female sibling mice of parental C57BL/ 6.NOD-Aec1Aec2 (P-DC) and C57BL/6.NOD-Aec1Aec2R(n) mice were injected with isoproterenol/pilocarpine, first at 8 weeks of age and then at 20 or 24 weeks of age, to stimulate saliva secretion. Saliva was collected from each mouse for 10 minutes starting 1 minute after injection of the secretagogue. The volume of each sample was measured and standardized relative to the weight of the mouse. Temporal reductions in saliva secretions, a marker for onset of clinical disease, were used to identify genetic regions containing genes necessary for development of salivary gland dysfunction and Sjögren syndrome. NS, not significant; RI, recombinant inbred.

euthanasia) and the sera were collected and tested on HEp-2 cells. As presented in Figure 5, a number of different patterns of ANA staining, including speckled/homogenous nuclear, cytoplasmic/nuclear membrane, speckled cytoplasm, and cytoplasmic staining, were detected in the sera from different $\mathrm{RI}$ lines. Cytoplasmic with nuclear membrane and cytoplasmic staining patterns appeared to be more prevalent in sera from SjS-nonsusceptible RI lines (RI02 and RI34), whereas sera from SjS-susceptible RI lines such as RI06C, RI09, and RI46B produced predominantly speckled/homogenous staining patterns. In general, a majority of sera from mice classified as SjSsusceptible RI lines produced ANA staining patterns observed with sera from parental C57BL/6.NOD-Aec1Aec2 mice and not NOD mice [9]. This difference between the C57BL/6 background-derived mice versus the NOD and NOD.B10. $\mathrm{H}^{\mathrm{b}}$ mice suggests that the ANA staining pattern is not diseasespecific and that the genetic background plays an important role in which ANAs are synthesized. Furthermore, the speckled pattern of staining in these RI lines appears to be characteristic of the staining observed with anti-SS-A/Ro and antiSS-B/La antibodies [30]. At the same time, the cytoplasmic punctate staining is characteristic of the staining observed with antibodies against GW bodies [31]. Confirmation of whether these antibodies are reactive with SS-A/Ro, SS-B/La, and/or GW bodies is currently ongoing.

\section{Redefining the Sjögren syndrome susceptibility Aec2 genetic region}

Based on the disease profiling data, we are now able to tentatively identify a small segment (subregion) of Aec2 containing genes essential and sufficient for development and onset of SjS-like disease associated with NOD and NOD-derived mice. As shown in Figure 6, the primary (or candidate) SjS susceptibility gene(s) on chromosome 1 lay within a genetic region around $79 \pm 5 \mathrm{cM}$. SjS susceptibility genes within this sublocus must be coexpressed with the NOD-derived genes of the Aec1 region of chromosome 3 in order to induce a clinical disease. Not surprisingly, however, this redefined Aec2 subregion contains multiple genes already shown to correlate with human and mouse $\mathrm{SjS}$ as well as several additional autoimmune diseases in mice. These genes provide a basis for developing hypothetical models of molecular mechanisms underlying SjS, as discussed below.

\section{Discussion}

In the present study, in which the specific goal was to redefine (and narrow) the boundaries of the Aec2 genetic region on chromosome 1 known to predispose NOD and NOD-derived lines of mice to $\mathrm{SjS}$, we generated a large set of new RI lines $(n=39)$ and examined each line for its SjS-like disease profile. Disease profiles obtained with the C57BL/6.NOD- 

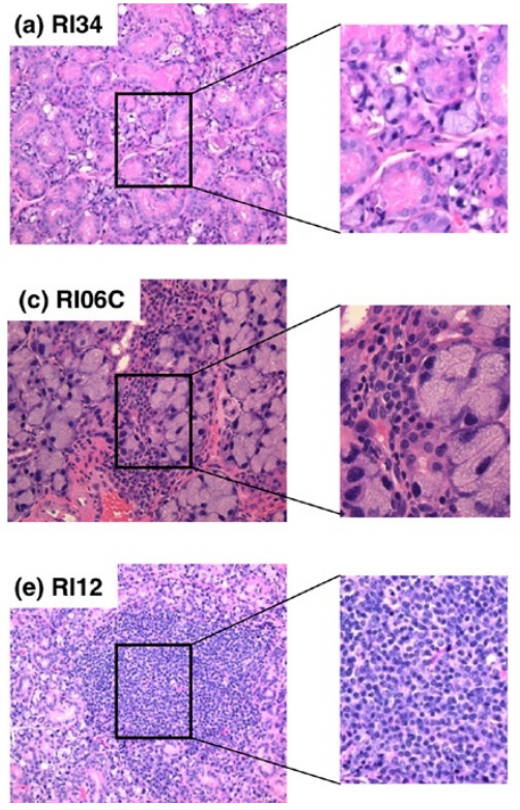
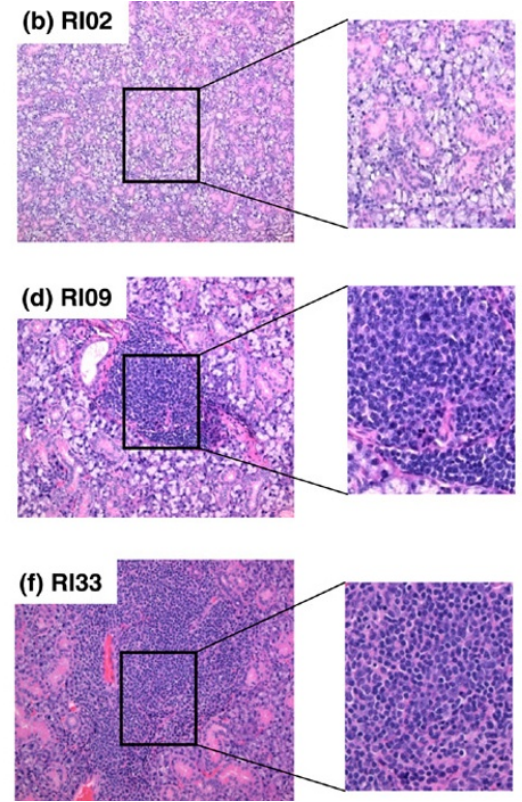

Histological characterization of sialadenitis of male and female C57BL/6.NOD-Aec1Aec2R(n) mice. Submandibular glands were freshly explanted from male and female C57BL/6.NOD-Aec1Aec2R $(n)$ mice euthanized at 20 or 24 weeks of age. The glands were fixed in $10 \%$ formalin, embedded in paraffin, and sectioned and stained with hematoxylin and eosin (H\&E) dye. Representative H\&E-stained histological sections of submandibular glands of selected recombinant inbred (RI) lines are presented: (a) RI34, (b) RI02, (c) RI06C, (d) RI09, (e) RI12, and (f) RI33. Original images were taken at $\times 100$ magnification, with inserts expanded to show structural detail.

Aec1Aec2R(n) Rl lines indicate that the Aec2 genetic region of C57BL/6.NOD-Aec1Aec2 mice, postulated to regulate primarily the pathophysiological and biochemical abnormalities that subsequently result in the activation of the autoimmune attack against the submandibular and lacrimal glands [10], is a single subregion mapping to the telomeric portion of chromosome 1 located at approximately $79 \pm 5 \mathrm{cM}$. However, penetrance and severity of SjS-like disease may be further influenced by genes located within a few centimorgans on the centromeric side of this region, possibly pointing to SjS-associated quantitative trait loci (QTL) genes. Although the size of the redefined $A e c 2$ region remains relatively large for identification of individual candidate SjS susceptibility genes, the genes residing within this subregion can be grouped into four functionally clustered sets, each suspected previously of involvement in SjS susceptibility. These are (a) endogenous viruses and oncogenic genes, (b) Fas/FasL-associated apoptosis, (c) $T_{H}$ 17-associated activities, and (d) fatty acid, lipid, lipoprotein, and cholesterol homeostasis. However, perhaps the most obvious aspect is the fact that this redefined Aec2 region contains the QTL-Ath1 region containing some 10 genes, including tumor necrosis factor ligand superfamily member 4 (Tnfsf4 or Ox4OL) and Tnfsf6 (Fasl).

Within the first set, several viral/oncogenic genes, such as Emv38 (endogenous ecotropic MuLV-38), Kras-2-rs1 (Kirsten rat sarcoma oncogene-2, related sequence-1), Xpr1 (xeno- tropic/polytropic retrovirus receptor-1), and Ab/2 (Abelson murine leukemia viral oncogene-2), are found in this redefined Aec2 subregion. In our earlier studies with NOD mice [12], we observed that high levels of interferon-gamma (INF- $\gamma$ ) were present in the salivary glands of neonate mice, suggesting an important role for INF- $\gamma$ in the delayed development/proliferation of acinar tissue observed in the salivary glands of neonate NOD mice. While it is logical to conclude that induction of INF$\gamma$ may be a result of short-term viral infection during the preterm and early postpartum periods, what might cause a viral outbreak at this time point remains unknown. It could be hypothesized that this occurs due to the changes in maternal hormone levels at this time. Perhaps more interesting, however, this region contains the gene Tnfsf6 encoding the proapoptotic protein FasL. FasL has numerous functions but is mainly involved in regulating immune responses, apoptosis, and retinal cell programmed death [4]. During the early phase 1 period of SjS-like disease in NOD mice, both FasL and Fas are upregulated at both the gene and protein levels, and this increased expression of Fas/FasL corresponds to the observed increase in acinar cell apoptosis within the glands [32]. However, it remains speculative whether there might be an association between endogenous/exogenous viral infection and Fas/FasL activity in the salivary and lacrimal glands.

The redefined Aec2 subregion also contains several genes involved in autoimmunity and/or tumorgenesis, the latter being 

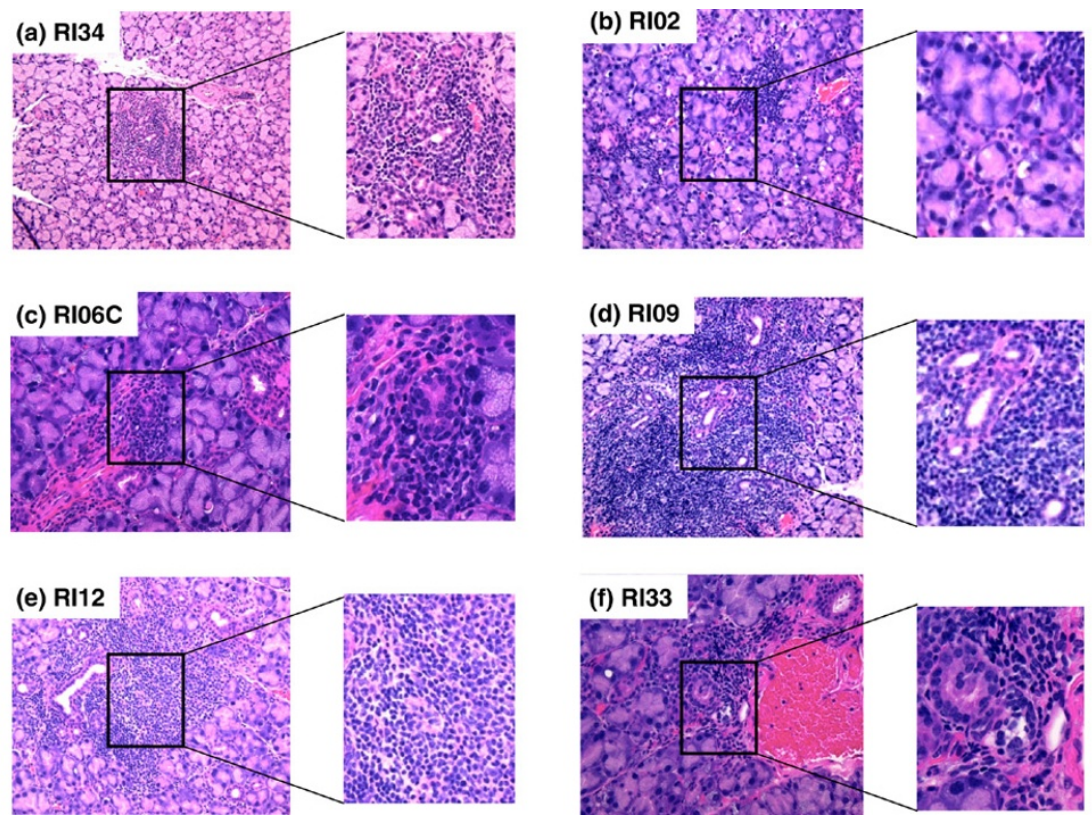

Histological characterization of dacryoadenitis of male and female C57BL/6.NOD-Aec1Aec2R(n) mice. Submandibular and lacrimal glands were freshly explanted from male and female C57BL/6.NOD-Aec1Aec2R(n) mice euthanized at 20 or 24 weeks of age. The glands were fixed in 10\% formalin, embedded in paraffin, and sectioned and stained with hematoxylin and eosin (H\&E) dye. Representative H\&E-stained histological sections of lacrimal glands of selected recombinant inbred (RI) lines are presented: (a) RI34, (b) RI02, (c) RI06C, (d) RI09, (e) RI12, and (f) RI33. Original images were taken at $\times 100$ magnification, with inserts expanded to show structural detail.

one clinical manifestation of SjS that occurs in a small subset of patients. Of interest, but not thought to be directly involved in the development and onset of SjS, is the presence of genes specific to the ocular/lacrimal gland etiology (for example, Pdc [phosducin], which is a protein of the retinal photoreceptors cells [33], and Myoc [myocilin], whose product interacts with olfactemedin involved in glaucoma [34]). However, whether any of these genes are related to SjS susceptibility and lacrimal gland disease or merely influence the secondary disease phenotypes often associated with SjS remains unknown. In

\section{Table 1}

Quantification of lymphocytic foci in the salivary and lacrimal glands of mice from several representative C57BL/6.NOD$\operatorname{Aec1Aec2R}(n)$ recombinant inbred lines

\begin{tabular}{|c|c|c|c|c|c|c|c|c|c|c|c|}
\hline \multirow[t]{3}{*}{ RI line } & \multirow[t]{3}{*}{ Age, weeks } & \multicolumn{5}{|c|}{ Submandibular glands } & \multicolumn{5}{|c|}{ Lacrimal glands } \\
\hline & & \multicolumn{2}{|c|}{ Number of mice } & \multicolumn{2}{|c|}{ Positive } & \multirow{2}{*}{$\begin{array}{c}\text { Average } \\
\text { number of LF }\end{array}$} & \multicolumn{2}{|c|}{ Number of mice } & \multicolumn{2}{|c|}{ Positive } & \multirow{2}{*}{$\begin{array}{c}\text { Average } \\
\text { number of LF }\end{array}$} \\
\hline & & Male & Female & Number & $\begin{array}{l}\text { Male and } \\
\text { female }\end{array}$ & & Male & Female & Number & $\begin{array}{l}\text { Male and } \\
\text { female }\end{array}$ & \\
\hline RI 34 & 20 and 24 & 6 & 7 & 3 & $23 \%$ & $1.0 \pm 0.0$ & 6 & 7 & 7 & $54 \%$ & $1.7 \pm 0.4$ \\
\hline RI 02 & 20 and 24 & 4 & 7 & 0 & $0 \%$ & $0.0 \pm 0.0$ & 6 & 8 & 4 & $29 \%$ & $1.5 \pm 0.3$ \\
\hline RI 06 & 20 & 4 & ND & 3 & $74 \%$ & $4.0 \pm 1.7$ & 4 & ND & 4 & $100 \%$ & $5.8 \pm 4.1$ \\
\hline RI 09 & 21 and 22 & 7 & 7 & 11 & $79 \%$ & $3.0 \pm 0.6$ & 7 & 7 & 6 & $43 \%$ & $2.8 \pm 0.7$ \\
\hline RI 12 & 24 & 7 & 4 & 5 & $45 \%$ & $2.4 \pm 0.7$ & 7 & 4 & 10 & $91 \%$ & $2.8 \pm 0.6$ \\
\hline RI 33 & 20 and 21 & 9 & 7 & 10 & $63 \%$ & $2.2 \pm 1.0$ & 9 & 7 & 13 & $81 \%$ & $6.5 \pm 4.5$ \\
\hline
\end{tabular}

LF, lymphocytic foci; ND, not done; RI, recombinant inbred. 


\begin{tabular}{|c|l|c|c|c|c|}
\hline \multicolumn{6}{|l|}{ Staining } \\
patterns
\end{tabular}

Detection of anti-nuclear autoantibodies in sera of C57BL/6.NOD-Aec1RAec2R(n) mice. Serum samples obtained from C57BL/6.NODAec1Aec2R(n) mice were diluted 1:40 and incubated with HEp-2 fixed substrate slides for 30 minutes at $25^{\circ} \mathrm{C}$ in a humidified chamber. The slides were then developed with Alexa 594-conjugated goat anti-mouse $\operatorname{lgG}$ and viewed by fluorescence microscopy at $\times 100$ magnification. Examples of speckled/homogenous staining of the nucleus (left panel), cytoplasmic/nuclear membrane staining (left center panel), speckled/cytoplasmic staining (right center panel), and cytoplasmic staining (right panel) were observed. The numbers of individual sera tested and the percentages of positive sera from a sampling of recombinant inbred (RI) lines exhibiting each of the patterns are listed. §Number of mice showing positive staining pattern over total. *Percentage of mice showing positive staining pattern. Aec, autoimmune exocrinopathy.

contrast, one genetic element in this region that has a direct association with the immunopathology of SjS is the QTL gene Cypr2 (cytokine production 2) [35]. CYPR-2 is known to regulate levels of interleukin-10 (IL-10), an important cytokine that enhances the activity of $B$ lymphocytes, and at the same time to regulate the functions of $T_{H} 1$ and $T_{H} 17$ cells [36]. Our recent microarray studies indicate that $/ / 10$ is not upregulated

Figure 6

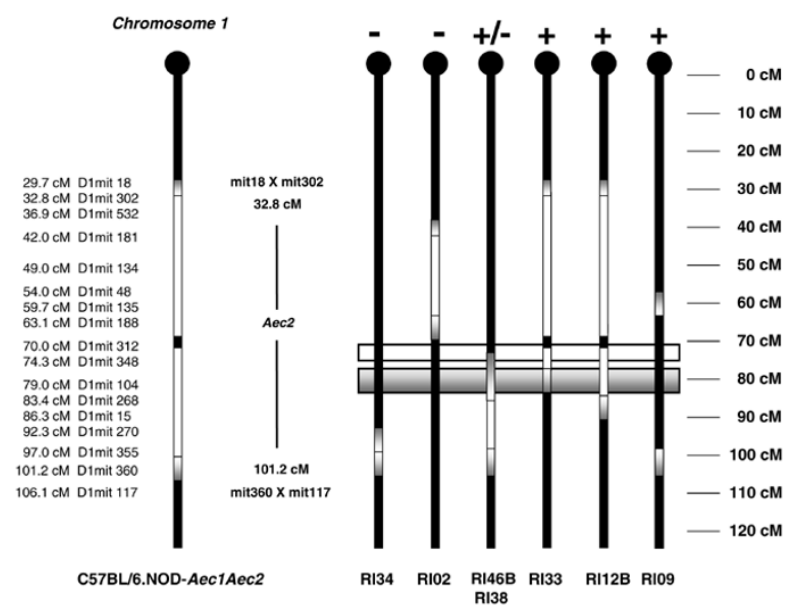

Redefining the boundaries for the Aec2 Sjögren Syndrome (SjS) susceptibility genetic locus. Based on the disease profiling of the C57BL/ 6.NOD-Aec1Aec2R $(n)$ recombinant inbred (RI) lines, the boundaries of the Aec2 genetic region containing SjS susceptibility genes have been temporarily reset to position $79 \pm 5 \mathrm{cM}$ of chromosome 1 (shaded gray gradient rectangular box). Possible quantitative trait loci genes may reside a few centimorgans centromeric to this region (unshaded rectangular box). during the development of $\mathrm{SjS}$ in the C57BL/6.NODAec1Aec2 mouse model [32], possibly indicating a lack of immune regulation by regulatory $T\left(T_{\text {reg }}\right)$ cells. If so, this lack of regulation by $\mathrm{IL}-10$ would be consistent with the results of gene therapy studies in which injections of vectors expressing recombinant $\mathrm{IL}-10$ reduced or suppressed clinical manifestations of SjS-like disease in both salivary and lacrimal glands of mice $[37,38]$.

Maintaining sufficient regulation of immune responses to prevent development of an overt autoimmunity is no doubt dependent on a physiological balance between $T_{H} 1, T_{H} 2$, $T_{H} 17$, and $T_{\text {reg }}$ cell interactions. This cellular interaction appears to be highly influenced by OX4OL encoded by the Tnfsf 4 gene and this gene is located within the redefined Aec2 region. $\mathrm{OX} 40 \mathrm{~L}$ is expressed by a number of distinct cell populations, including activated dendritic cells [39]. OX4OL is capable of functioning as an inhibitor of the maturation of $\mathrm{T}_{\text {reg }} 1$ cells [40], a regulatory cell population normally producing IL10 and INF- $\gamma$, which (in conjunction with IL-27) can inhibit the effector $C D 4+T_{H} 17$ cells [41]. Reduced $\mathrm{T}_{\text {reg }} 1$ cell function, therefore, results in a positive feedback for the generation/activation of effector CD4 $+T_{H} 17$ cells (Figure 7). These effector $\mathrm{T}_{\mathrm{H}} 17$ cells produce predominantly IL-17, IL-21, and IL-22 plus factors like nitric oxide, matrix metalloproteinase, and prostaglandin $E_{2}$, each of which is shown to play an important role in the immunopathophysiology of several autoimmune diseases, including SjS [41]. Thus, we hypothesize that the presence of $\mathrm{T}_{\mathrm{H}} 17$ cells in the salivary and lacrimal glands of $\mathrm{C} 57 \mathrm{BL} /$ 6.NOD-Aec1Aec2 mice, as well as SjS patients [42], indicates an imbalance in the $T_{H} 17 / T_{\text {reg }} 1$ ratio favoring the $T_{H} 17$ population(s). A recent study indicates that retinoic acid can facilitate an increase in the numbers of Foxp $3^{+} T_{\text {reg }}$ cells and 


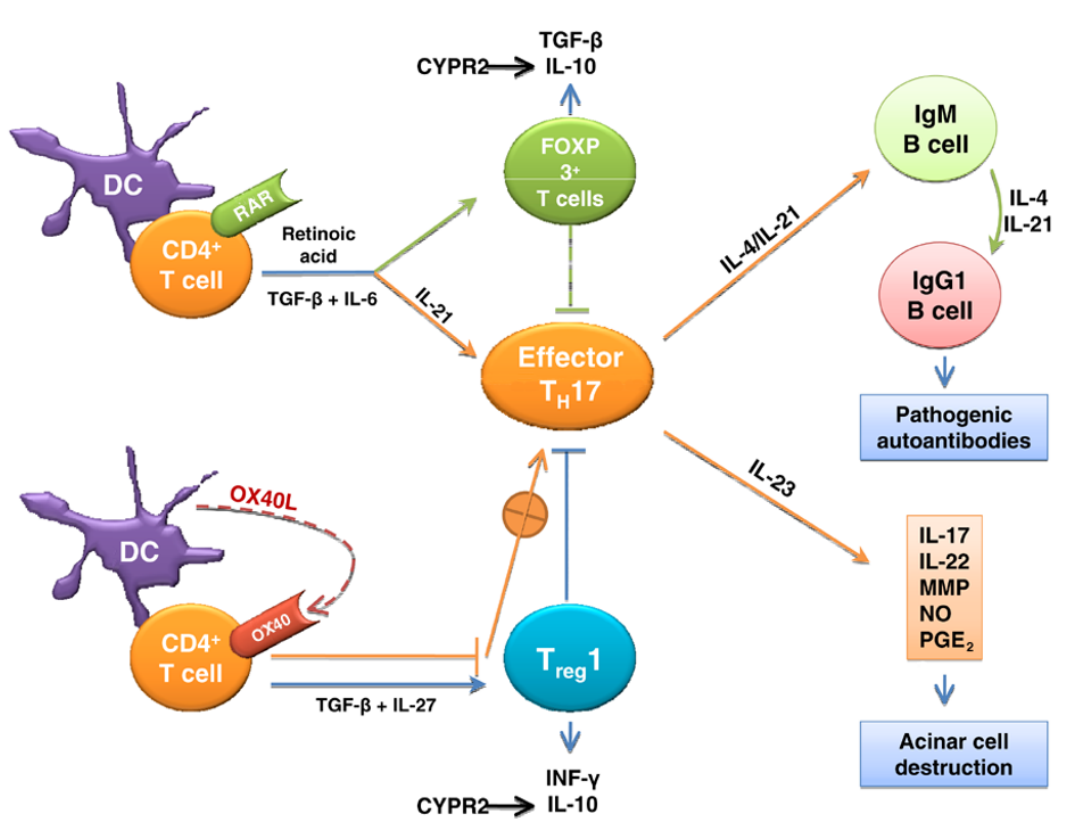

Proposed model for how OX40L:OX40 promotes autoimmunity in Sjögren syndrome-like disease of C57BL/6.NOD-Aec1Aec2 mice. Cellular interactions involved in the development of an autoimmune response against the salivary and lacrimal glands leading to loss of acinar tissue are presented. DC, dendritic cell; IL, interleukin; INF- $\gamma$, interferon-gamma; MMP, matrix metalloproteinase; NO, nitric oxide, PGE ${ }_{2}$, prostaglandin $E_{2} ;$ TGF- $\beta$, transforming growth factor-beta; $T_{\text {reg}}$, regulatory $T$ (cell).

simultenously inhibit the formation of effector $T_{H} 17$ cells [43]. Interestingly, we have found that expression of the retinoic receptors, $\operatorname{Rxr}$ (retinoid $\times$ receptor) and $\operatorname{Rar}$ (retinoic acid receptor), is downregulated in the lacrimal glands of C57BL/ 6.NOD-Aec1Aec2 mice [44]. This observation is again consistent with a potential problem in cellular homeostasis, especially at the level of macrophages, dendritic cells, and even production of the FOXP3 ${ }^{+} \mathrm{T}_{\text {reg }}$ cell populations whose differentiation and functional maturation are highly dependent on retinoic and fatty acid stimulation. As presented in Figure 7, there is a reciprocal maturation of effector $C D 4{ }^{+} T_{H} 17$ and $\mathrm{FOXP}^{+} \mathrm{T}_{\text {reg }}$ cells dependent on the relative balance of IL- 6 and transforming growth factor-beta influenced by retinoic acid. Thus, the intricacy and balance between OX4OL, the retinoids, proinflammatory cytokines, and development of $T_{\text {reg }}$ cells appear to impact the potential development and onset of autoimmunity, which in SjS appears to favor activation of effector $\mathrm{T}_{\mathrm{H}} 17$ cells.

Although several factors encoded by genes in the redefined Aec2 region may be involved in secondary manifestations of $\mathrm{SjS}, \mathrm{OX} 4 \mathrm{OL}$ is the one factor that clearly stands out as a primary candidate gene underlying not only the recognized immune dysregulation, as presented above, but also the pathophysiological aberrations associated with chromosome 1 of the C57BL/6.NOD-Aec1Aec2 SjS model. In this redefined region, a common functional cluster of lipid, lipoprotein, cholesterol, and fatty acid regulatory and processing elements is found, including Hdlq14 (high-density lipoprotein QLT-14), Hdlq5 or Apoa2 (high-density lipoprotein QTL-5), Gpa33 (glycoprotein A33), Cq1 (cholesterol OTL-1), Prdx6 (peroxiredoxin), and (of special note) Soat1 (sterol O-acyltransferase1). Involvement of lipids and fatty acids in the pathology of $S j S$ has become a major focus of SjS research as lipid depositions [45] and changes in lipid rafts [46] appear to influence the pathology in both salivary and lacrimal glands. Furthermore, our recent genomic microarray studies - $[44,47]$ (C.O. Nguyen, S. Ashok, R.A McIndoe, J.X. She, B.H. Lee, A.B. Peck, unpublished data) - indicate that multiple genes involved in fatty acid, lipid, lipoprotein, and cholesterol homeostasis/ transport are differentially expressed, corresponding with lipid deposits, dysfunctional dendritic cells, and onset of autoimmunity. As illustrated in Figure 8, various relationships between genes controlling free fatty acid, lipid, and lipoprotein homeostasis are directly or indirectly dependent on the activities of OX4OL. As a consequence, imbalances in this homeostasis regulated in part by OX40/OX40L can result in widespread pathology, including inflammation and cell death. Based on differential gene expression data, there are major reductions in the levels of transcripts encoding FDFT-1 (farnesyl diphosphate farnesyl transferase-1), ABCA1 (ATP-binding cassette, subfamily $A$ [ABC1] member 1 ), and the retinoic acid receptors, RRX and RAR. At the same time, increased levels of transcripts encoding the low- and high-density lipoprotein 
Figure 8

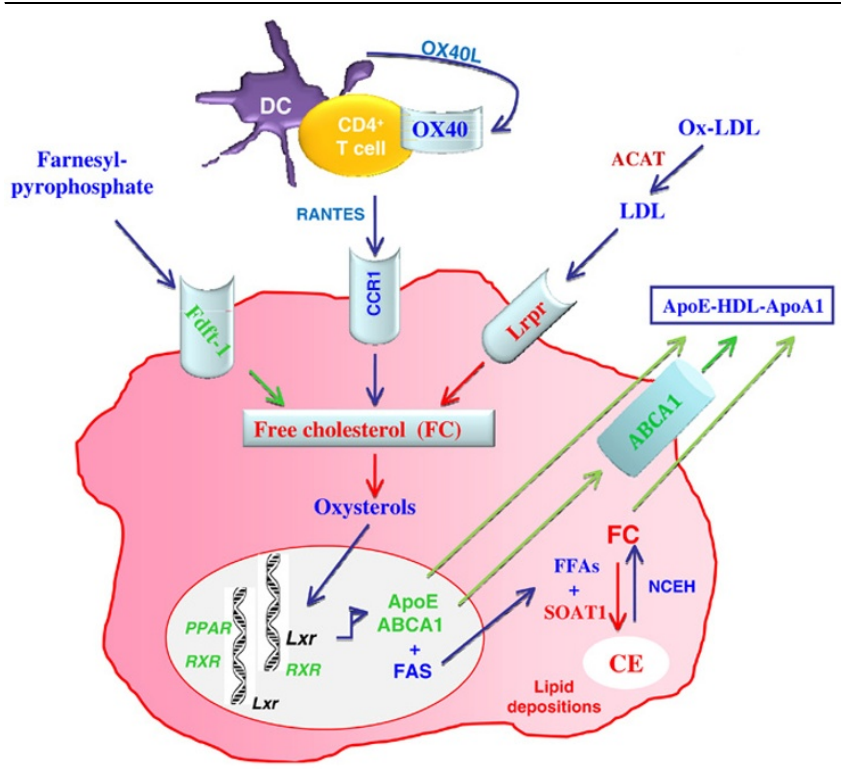

Proposed genetic predisposition for dysregulated homeostasis/transport of lipid, lipoprotein, cholesterol, and fatty acid metabolism leading to lipid depositions in the salivary and lacrimal glands of C57BL/ 6.NOD-Aec1Aec2 mice and Sjögren syndrome patients. Accumulation of free cholesterols (FCs) inside the cells resulted from increased uptake of low- and high-density lipid receptors. In addition, impairment of ABCA1 membrane transporter leads to the accumulation of cholesteryl esters (CEs) metabolized by sterol O-acyltransferase-1 (SOAT-1) using FCs and free fatty acids (FFAs). ABCA1, ATP-binding cassette, subfamily $A[A B C 1]$ member $1 ; A C A T$, acyl-coenzyme $A$ : cholesterol acyltransferase; ApoE, apolipoprotein E; DC, dendritic cell; Fdft-1, farnesyl diphosphate farnesyl transferase-1; HDL, high-density lipid; LDL, low-density lipid; Lrpr, low-density lipid-related protein receptor; NCEH, neutral cholesterol esters hydrolase; Ox-LDL, oxidized low-density lipid; PPAR, peroxisome proliferator activated receptor; RANTES, regulated on activation normal T cell expressed and secreted; RXR, retinoid $X$ receptor. Adapted from [48].

receptors, as well as SOAT-1, are observed. We hypothesize, therefore, that an imbalance occurs in the production of cholesterol and the increased level of cholesterol results in greater amounts being converted by SOAT- 1 to cholesteryl esters that accumulate within the cells due to the downregulation or dysfunction of the lipid transporter. In addition, the functional activities of cells whose differentiation and maturation are dependent on the retinoids and the RXR/RAR-PPAR (RXR/ RAR-peroxisome proliferator activated receptor-gamma) signaling pathways (for example, macrophages and dendritic and FOXP3 ${ }^{+} \mathrm{T}$ cells) will be altered due to altered development, ultimately affecting antigen presentation and balanced production of regulatory cytokines.

\section{Conclusion}

Identifying gene products that are differentially expressed in the Aec2 SjS susceptibility subregion defined by the new RI lines is moving us closer to identifying specific candidate genes involved in the onset and development of SjS-like dis- ease. Based on our current data, our focus is turning to $\mathrm{O} \times 4 \mathrm{OL}$ as an effective candidate gene for the development of SjS. The future application of genetic knockout mice and/or short interfering RNA will permit us to further our understanding of the potential role of $\mathrm{O} x 40 \mathrm{~L}$ in SjS and, more importantly, to translate its relevancy to human $\mathrm{SjS}$.

\section{Competing interests}

The authors declare that they have no competing interests.

\section{Authors' contributions}

ABP performed the genotyping of mice and assisted with the study design and manuscript preparation. JT carried out histological analysis. JGC, LC, and JN performed saliva collections and disease profilings of the mice. $\mathrm{BHL}$ helped with manuscript preparation. CQN participated in the design of the study, ANA staining, saliva collections, data analyses, and manuscript preparation. All authors read and approved the final manuscript.

\section{Acknowledgements}

We thank Robert Haynes for the countless hours spent in caring for and maintaining precise records of these RI lines and Sung Kim for his help in analyzing the function and pathway associations of selected genes. This work was supported in part by Public Health Service (PHS) grant DE014344 from the National Institutes of Health (to ABP) and by the Center for Orphaned Autoimmune Disorders at the University of Florida. CON was supported by a postdoctoral fellowship from PHS grant T32 DE07200.

\section{References}

1. Fox RI, Kang HI: Pathogenesis of Sjögren's syndrome. Rheum Dis Clin North Am 1992, 18:517-538.

2. Hansen A, Lipsky PE, Dorner T: New concepts in the pathogenesis of Sjogren syndrome: many questions, fewer answers. Curr Opin Rheumatol 2003, 15:563-570.

3. Jonsson R, Haga HJ, Gordon TP: Current concepts on diagnosis, autoantibodies and therapy in Sjögren's syndrome. Scand $J$ Rheumatol 2000, 29:341-348.

4. Nguyen CQ, Cha SR, Peck AB: Sjögren's syndrome (SjS)-like disease of mice: the importance of $B$ lymphocytes and autoantibodies. Front Biosci 2007, 12:1767-1789.

5. Makino S, Kunimoto K, Muraoka Y, Mizushima Y, Katagiri K, Tochino Y: Breeding of a non-obese, diabetic strain of mice. Jikken Dobutsu 1980, 29:1-13.

6. Konttinen YT, Tensing EK, Laine M, Porola P, Tornwall J, Hukkanen $\mathrm{M}$ : Abnormal distribution of aquaporin-5 in salivary glands in the NOD mouse model for Sjögren's syndrome. J Rheumatol 2005, 32:1071-1075.

7. Rosignoli F, Roca V, Meiss R, Leceta J, Gomariz RP, Perez Leiros $\mathrm{C}$ : Defective signalling in salivary glands precedes the autoimmune response in the non-obese diabetic mouse model of sialadenitis. Clin Exp Immunol 2005, 142:411-418.

8. Humphreys-Beher MG, Hu Y, Nakagawa Y, Wang PL, Purushotham KR: Utilization of the non-obese diabetic (NOD) mouse as an animal model for the study of secondary Sjögren's syndrome. Adv Exp Med Biol 1994, 350:631-636.

9. Nguyen C, Singson E, Kim JY, Cornelius JG, Attia R, Doyle ME, Bulosan M, Cha S, Peck AB: Sjögren's syndrome-like disease of C57BL/6.NOD-Aec1 Aec2 mice: gender differences in keratoconjunctivitis sicca defined by a cross-over in the chromosome 3 Aec1 locus. Scand J Immuno/ 2006, 64:295-307.

10. Brayer J, Lowry J, Cha S, Robinson CP, Yamachika S, Peck AB, Humphreys-Beher MG: Alleles from chromosomes 1 and 3 of NOD mice combine to influence Sjögren's syndrome-like autoimmune exocrinopathy. J Rheumatol 2000, 27:1896-1904. 
11. Brayer JB, Cha S, Nagashima $H$, Yasunari U, Lindberg A, Diggs $S$, Martinez J, Goa J, Humphreys-Beher MG, Peck AB: IL-4-dependent effector phase in autoimmune exocrinopathy as defined by the NOD.IL-4-gene knockout mouse model of Sjögren's syndrome. Scand J Immunol 2001, 54:133-140.

12. Cha S, Brayer J, Gao J, Brown V, Killedar S, Yasunari U, Peck AB: A dual role for interferon-gamma in the pathogenesis of Sjögren's syndrome-like autoimmune exocrinopathy in the nonobese diabetic mouse. Scand J Immuno/ 2004, 60:552-565.

13. Cha $S$, Peck $A B$, Humphreys-Beher MG: Progress in understanding autoimmune exocrinopathy using the non-obese diabetic mouse: an update. Crit Rev Oral Biol Med 2002, 13:5-16.

14. Cha S, van Blockland SC, Versnel MA, Homo-Delarche $F$, Nagashima H, Brayer J, Peck AB, Humphreys-Beher MG: Abnormal organogenesis in salivary gland development may initiate adult onset of autoimmune exocrinopathy. Exp Clin Immunogenet 2001, 18:143-160.

15. Gao J, Cha S, Jonsson R, Opalko J, Peck AB: Detection of antitype 3 muscarinic acetylcholine receptor autoantibodies in the sera of Sjögren's syndrome patients by use of a transfected cell line assay. Arthritis Rheum 2004, 50:2615-2621.

16. Gao J, Killedar S, Cornelius JG, Nguyen C, Cha S, Peck AB: Sjögren's syndrome in the NOD mouse model is an interleukin-4 time-dependent, antibody isotype-specific autoimmune disease. J Autoimmun 2006, 26:90-103.

17. Robinson CP, Brayer J, Yamachika S, Esch TR, Peck AB, Stewart $C A$, Peen $E$, Jonsson R, Humphreys-Beher MG: Transfer of human serum IgG to nonobese diabetic Igmu null mice reveals a role for autoantibodies in the loss of secretory function of exocrine tissues in Sjögren's syndrome. Proc Natl Acad Sci USA 1998, 95:7538-7543.

18. Robinson $\mathrm{CP}$, Cornelius J, Bounous $\mathrm{DI}$, Yamamoto $\mathrm{H}$, HumphreysBeher MG, Peck AB: Infiltrating lymphocyte populations and cytokine production in the salivary and lacrimal glands of autoimmune NOD mice. Adv Exp Med Biol 1998, 438:493-497.

19. Robinson CP, Yamamoto $H$, Peck AB, Humphreys-Beher MG: Genetically programmed development of salivary gland abnormalities in the NOD (nonobese diabetic)-scid mouse in the absence of detectable lymphocytic infiltration: a potential trigger for sialoadenitis of NOD mice. Clin Immunol Immunopathol 1996, 79:50-59.

20. Robinson CP, Yamachika S, Bounous DI, Brayer J, Jonsson R, Holmdahl R, Peck AB, Humphreys-Beher MG: A novel NOD-derived murine model of primary Sjögren's syndrome. Arthritis Rheum 1998, 41:150-156.

21. Cha S, Nagashima H, Brown VB, Peck AB, Humphreys-Beher MG: Two NOD Idd-associated intervals contribute synergistically to the development of autoimmune exocrinopathy (Sjögren's syndrome) on a healthy murine background. Arthritis Rheum 2002, 46:1390-1398.

22. Brayer JB, Humphreys-Beher MG, Peck AB: Sjögren's syndrome: immunological response underlying the disease. Arch Immunol Ther Exp (Warsz) 2001, 49:353-360.

23. Prochazka M, Leiter EH, Serreze DV, Coleman DL: Three recessive loci required for insulin-dependent diabetes in nonobese diabetic mice. Science 1987, 237:286-289.

24. Morel L, Rudofsky UH, Longmate JA, Schiffenbauer J, Wakeland EK: Polygenic control of susceptibility to murine systemic lupus erythematosus. Immunity 1994, 1:219-229.

25. Vitali C, Bombardieri S, Jonsson R, Moutsopoulos HM, Alexander EL, Carsons SE, Daniels TE, Fox PC, Fox RI, Kassan SS, Pillemer SR, Talal N, Weisman MH: Classification criteria for Sjögren's syndrome: a revised version of the European criteria proposed by the American-European Consensus Group. Ann Rheum Dis 2002, 61:554-558.

26. Pourmand N, Wahren-Herlenius M, Gunnarsson I, Svenungsson E, Lofstrom B, loannou $Y$, Isenberg DA, Magnusson CG: Ro/SSA and $\mathrm{La} / \mathrm{SSB}$ specific IgA autoantibodies in serum of patients with Sjögren's syndrome and systemic lupus erythematosus. Ann Rheum Dis 1999, 58:623-629.

27. Maran R, Dueymes M, Pennec YL, Casburn-Budd R, Shoenfeld Y, Youinou P: Predominance of IgG1 subclass of anti-Ro/SSA, but not anti-La/SSB antibodies in primary Sjögren's syndrome. J Autoimmun 1993, 6:379-387.

28. Aparisi L, Farre A, Gomez-Cambronero L, Martinez J, De Las Heras G, Corts J, Navarro S, Mora J, Lopez-Hoyos M, Sabater L, Ferran$\operatorname{dez}$ A, Bautista D, Perez-Mateo M, Mery S, Sastre J: Antibodies to carbonic anhydrase and IgG4 levels in idiopathic chronic pancreatitis: relevance for diagnosis of autoimmune pancreatitis. Gut 2005, 54:703-709.

29. Harley JB, Alexander EL, Bias WB, Fox OF, Provost TT, Reichlin M, Yamagata H, Arnett FC: Anti-Ro (SS-A) and anti-La (SS-B) in patients with Sjögren's syndrome. Arthritis Rheum 1986, 29:196-206.

30. Pollock $\mathrm{W}$, Toh $\mathrm{BH}$ : Routine immunofluorescence detection of Ro/SS-A autoantibody using HEp-2 cells transfected with human 60 kDa Ro/SS-A. J Clin Pathol 1999, 52:684-687.

31. Stinton LM, Eystathioy T, Selak S, Chan EK, Fritzler MJ: Autoantibodies to protein transport and messenger RNA processing pathways: endosomes, lysosomes, Golgi complex, proteasomes, assemblyosomes, exosomes, and GW bodies. Clin Immunol 2004, 110:30-44.

32. Kong L, Robinson CP, Peck AB, Vela-Roch N, Sakata KM, Dang $H$, Talal N, Humphreys-Beher MG: Inappropriate apoptosis of salivary and lacrimal gland epithelium of immunodeficient NOD-scid mice. Clin Exp Rheumatol 1998, 16:675-681.

33. Schulz R: The pharmacology of phosducin. Pharmacol Res 2001, 43:1-10.

34. Gobeil S, Letartre L, Raymond V: Functional analysis of the glaucoma-causing TIGR/myocilin protein: integrity of amino-terminal coiled-coil regions and olfactomedin homology domain is essential for extracellular adhesion and secretion. Exp Eye Res 2006, 82:1017-1029.

35. Llorente L, Richaud-Patin Y, Fior R, Alcocer-Varela J, Wijdenes J, Fourrier BM, Galanaud P, Emilie D: In vivo production of interleukin-10 by non-T cells in rheumatoid arthritis, Sjögren's syndrome, and systemic lupus erythematosus. A potential mechanism of B lymphocyte hyperactivity and autoimmunity. Arthritis Rheum 1994, 37:1647-1655.

36. Yoshihara K, Yamada H, Hori A, Yajima T, Kubo C, Yoshikai Y: IL15 exacerbates collagen-induced arthritis with an enhanced CD4+ T cell response to produce IL-17. Eur J Immunol 2007 37:2744-2752.

37. Kok MR, Yamano S, Lodde BM, Wang J, Couwenhoven RI, Yakar S, Voutetakis A, Leroith D, Schmidt M, Afione S, Pillemer SR, Tsutsui MT, Tak PP, Chiorini JA, Baum BJ: Local adeno-associated virus-mediated interleukin 10 gene transfer has disease-modifying effects in a murine model of Sjögren's syndrome. Hum Gene Ther 2003, 14:1605-1618.

38. Zhu Z, Stevenson D, Schechter JE, Mircheff AK, Ritter T, Labree L, Trousdale MD: Prophylactic effect of IL-10 gene transfer on induced autoimmune dacryoadenitis. Invest Ophthalmol Vis Sci 2004, 45:1375-1381.

39. Baum PR, Gayle RB 3rd, Ramsdell F, Srinivasan S, Sorensen RA, Watson ML, Seldin MF, Baker E, Sutherland GR, Clifford KN, Alderson MR, Goodwin RG, Fanslow WC: Molecular characterization of murine and human OX40/OX40 ligand systems: identification of a human OX40 ligand as the HTLV-1-regulated protein gp34. EMBO J 1994, 13:3992-4001.

40. Ito $\mathrm{T}$, Wang $\mathrm{YH}$, Duramad $\mathrm{O}$, Hanabuchi $\mathrm{S}$, Perng OA, Gilliet $\mathrm{M}$, Oin FX, Liu YJ: OX40 ligand shuts down IL-10-producing regulatory T cells. Proc Natl Acad Sci USA 2006, 103:13138-13143.

41. Bettelli E, Korn T, Oukka M, Kuchroo VK: Induction and effector functions of $\mathrm{T}(\mathrm{H}) 17$ cells. Nature 2008, 453:1051-1057.

42. Nguyen CQ, Hu MH, Li Y, Stewart C, Peck AB: Salivary gland tissue expression of interleukin-23 and interleukin-17 in Sjögren's syndrome: findings in humans and mice. Arthritis Rheum 2008, 58:734-743.

43. Xiao S, Jin H, Korn T, Liu SM, Oukka M, Lim B, Kuchroo VK: Retinoic acid increases Foxp $3^{+}$regulatory $T$ cells and inhibits development of Th17 cells by enhancing TGF-beta-driven Smad3 signaling and inhibiting IL-6 and IL-23 receptor expression. $J$ Immunol 2008, 181:2277-2284.

44. Nguyen CQ, Ashok S, She JX, Mclndoe RA, Peck AB: Differential gene expressions in the lacrimal gland during development and onset of keratoconjunctivitis sicca in Sjögren's syndrome (SjS)-like disease of the C57BL/6.NOD-Aec1Aec2 mouse. Exp Eye Res in press.

45. Lodde BM, Sankar V, Kok MR, Leakan RA, Tak PP, Pillemer SR: Serum lipid levels in Sjögren's syndrome. Rheumatology (Oxford) 2006, 45:481-484.

46. Ishikawa Y, Cho G, Yuan Z, Inoue N, Nakae Y: Aquaporin-5 water channel in lipid rafts of rat parotid glands. Biochim Biophys Acta 2006, 1758:1053-1060. 
Arthritis Research \& Therapy Vol 10 No 6 Nguyen et al.

47. Killedar SY, Eckenrode SE, Mclndoe RA, She JX, Nguyen CQ, Peck $A B$, Cha SR: Early pathogenic events associated with Sjögren's syndrome (SjS)-like disease of the nod mouse using microarray analysis. Lab Invest 2006, 86:1243-1260.

48. Li AC, Glass CK: The macrophage foam cell as a target for therapeutic intervention. Nat Med 2002, 8:1235-1242. 